\title{
A qualitative study exploring the effects of participation in a community-based pre-operative exercise programme on perceived wellbeing and quality of life in people with newly diagnosed prostate cancer: A pilot study
}

Lisa Loughney ( $\square$ lisa.loughney@gmail.com )

Dublin City University

Rachel McGowan

National University Galway

Kiaran O'Malley

Mater Misericordiae University Hospital

Noel McCaffrey

ExWell Medical

Brona Furlong

Waterford Institute of Technology

Deirdre Walsh

Athlone Institute of Technology

\section{Research article}

Keywords: surgical-oncology, prostate cancer, community, pre-operative exercise training, quality of life, wellbeing

Posted Date: December 6th, 2019

DOI: https://doi.org/10.21203/rs.2.18345/v1

License: (c) (i) This work is licensed under a Creative Commons Attribution 4.0 International License.

Read Full License 


\section{Abstract}

Purpose: People with a newly diagnosed prostate cancer are often treated by surgery. The time window between cancer diagnosis and surgery causes high levels of uncertainty and stress, which negatively impact quality of life (QoL). This study explored the experience of participating in a community-based pre-operative exercise programme (in the time between cancer diagnosis and surgery) and its impact on perceived wellbeing and QoL in people with newly diagnosed prostate cancer.

Methods: Eleven men diagnosed with a new prostate cancer diagnosis were recruited and undertook a prescribed community-based pre-operative exercise training programme in the time available prior to surgery. Following completion of the pre-operative exercise programme (within 1 week before surgery), participants took part in one semi-structured interview which explored four broad QoL domains: physical; psychological; social; and spiritual wellbeing. Data were analysed using thematic analysis (a bottom up/inductive analysis).

Results: Data supported four main themes. Participation in the community-based pre-operative exercise training programme (over a mean (SD) of 4 (2) weeks) provided participants with: 1) a teachable moment; 2) a journey of preparation; 3) provided them with a sense of optimism; and 4) social connectedness.

Conclusion: This community-based pre-operative exercise training programme enhanced wellbeing and served as an opportunity to improve perceived QoL. Further research is required to explore this in a larger sample.

\section{Background}

Prostate cancer is the second most common cancer in men and the fourth most common worldwide [1]. In Ireland, it is the second most commonly diagnosed cancer [2]. Twenty-eight percent of people with a newly diagnosed prostate cancer are treated by surgery and hormone therapy and $36 \%$ are treated by radiotherapy [3]. Prostate cancer treatment can have many adverse effects such as cause sexual dysfunction, urinary and bowel problems, fatigue, distress and anxiety [4].

The waiting time between a cancer diagnosis and surgery can cause high levels of uncertainty, anxiety and distress [5-6]. Enhancing quality of life (QoL) is a key area of healthcare, whereby "health" encompasses not only the absence of disability and disease but also a sense of wellbeing [7]. Physical activity greatly enhances wellbeing and QoL in cancer survivors across several domains such as physical (i.e. managing the physical consequences of cancer and its treatment), psychological (i.e. evoking positive self-perceptions), social (i.e. feeling understood by others), and spiritual (i.e. redefining life purpose) [8]. Additionally, physical activity alleviates symptoms and assists recovery [9-10]. To date, exercise studies for people with prostate cancer that explore wellbeing [11] and QoL [12-13] have been reported during cancer treatment. To our knowledge no studies have been reported in the pre-operative setting. Therefore, the aim of this study was to explore the experience of participating in a community- 
based pre-operative exercise programme and its subsequent impact on perceived wellbeing and QoL in people with a newly diagnosed prostate cancer who are scheduled for surgery.

\section{Methods}

\section{Study design}

This study is a sub-study, part of a larger pre-post intervention pilot study, which investigated the adherence, compliance and effectiveness of a community-based pre-operative exercise training programme on health-related (HR) components of fitness and health-related quality of life (HRQoL) (in press with Perioperative Medicine). The study was approved by Dublin City University Research Ethics Committee (REC/2015/207) with patients referred from the Mater Misericordiae University Hospital, Ireland. The study flow diagram is presented in Figure 1.

\section{Participants}

Inclusion criteria included aged $\geq 18$ years with prostate cancer scheduled for surgery. The exclusion criteria included contraindications to exercise including uncontrolled cardiovascular conditions, significant skeletal muscle, orthopaedic, or neurological condition, or cognitive decline, significant mental illness or intellectual disability that prevented participation in a physical training programme. Eligible patients with a new prostate cancer diagnosis scheduled for surgery were given a patient information leaflet by either their surgical consultant or clinical nurse specialist in the outpatient cancer clinic. Interested patients made contact or were contacted by the lead study clinical exercise physiologist (LL) (dependent on outpatient clinic). Written informed consent was provided at the baseline visit. Participant medical co-morbidity and medication list were obtained at this visit.

\section{Community-based exercise intervention}

MedEx (now rebranded as ExWell Medical) is an established community-based, medically supervised, chronic illness rehabilitation programme based in the gym at Dublin City University campus [20]. The exercise intervention for this study was delivered as part of the MedEx programme. Participants were offered and selected from a range of community-based intervention options including either a centrebased exercise programme (CBEP) or a home-based exercise programme (HBEP). The variety of community options aimed to facilitate equality of access to eligible participants. The HBEP catered for participants living outside Dublin or those that could not access the centre.

The CBEP involved attending the MedEx centre at 10 am, any week day, from Monday to Friday. Participants undertook exercise training as part of the cancer prepare service which includes combination of people with newly diagnosed cancers and attendance at sessions can vary from eight to twelve people per session. The cost for the CBEP was $€ 7 / 8$ (with/without a medical card) per session or $€ 45 / 50$ (with/without a medical card) per month (parking was included in this payment). Participants on the 
CBEP were encouraged to be physically active outside of the programme but no formal exercise advice or record log sheets were provided.

The HBEP involved undertaking exercises independently and the cost of the HBEP was a standalone fee of $€ 15$. Participants on the HBEP were assessed during the same time the CBEP was being delivered and were integrated among the CBEP group during their visit. The HBEP was prescribed at baseline assessment and involved educating the participants on aerobic and resistance exercises. All participants undertook a 10 minute exercise session on the cycle ergometer, under the supervision of the clinical exercise physiologist (LL), to provide participants with an understanding of what level of intensity they should be aiming to achieve with their aerobic exercise at home, guided by the rate of perceived exertion (RPE) scale. All participants were instructed on how to complete resistance exercises in a safe manner (i.e. weight selection, technique, breathing, rest periods). All participants on the HBEP were provided with their exercise prescription, a RPE scale and a log sheet to record exercise sessions. They were contacted by telephone on a weekly basis (ideally same time and day each week) to review and feedback on progress, to provide continued motivation and to provide participants an opportunity to ask questions.

The CBEP and HBEP exercise prescription for interval (moderate to high intensity) exercise training were similar and are described using by the FITT-P (frequency, intensity, time, type, progression) principle (Table 1). However, the high intensity exercise training was only prescribed to certain individuals who could tolerate the interval training very well and only to participants on the CEBP. Adherence to the CBEP was assessed by number of sessions attended, recorded by the lead researcher (LL). Adherence to the HBEP was self-reported by the participant using a log diary.

\section{Procedure}

Semi-structured individual interviews were conducted in the MedEx centre at Dublin City University following completion of the pre-operative exercise training programme, within 1 week prior to surgery. Interviews were a standard part of the pilot study procedure for all participants. Informed consent regarding interview participation was discussed and agreed at the baseline visit prior to the beginning of the intervention. An audio recorder and a quiet room were used for the interviews. A semi-structured interview guide was developed to explore four domains of wellbeing and QoL: social; spiritual; psychological; and physical, which are in accordance with Ferrell et al., 1995 [15]. The interview guide consisted of open-ended questions concentrating on the participants' experience of participation in the exercise programme as well as their current perception of their wellbeing and QoL. Participants were encouraged to lead the flow of the interview while non-directive general probes were used in order to follow up on topics. The interview guide and prompts used for the interviews are presented in supplementary Appendix 1.

\section{Data Analysis}

All interviews were conducted, transcribed and analysed by authors (LL \& RMcG). Interviews were transcribed verbatim and checked for accuracy through multiple listenings within the thematic analysis 
process. Thematic analysis was used to analyse the data using the following six steps [16]. First, repeated listenings and readings of the transcripts was carried out to become familiar with the data and to gain a deeper understanding. Second, the coding phase took place which involved actively reading the data, taking note of any relevant patterns or meanings within the data. Initial coding was conducted in order to identify any significant ideas or patterns which were thought to be important or relevant. Third, was the generation of themes, which involved synthesising the codes to accurately reflect the relevant over-arching themes within the data forming a thematic map of the interview data. Fourth, was a review of each theme where all relevant codes and illustrative quotations were reviewed to ensure coherency to the main scope of the relevant theme. It was necessary here to reconsider each theme and its validity and value to the overall research question (i.e., exploring the experience of participation in a community-based pre-operative exercise programme, in the time between cancer diagnosis and surgery, and subsequent impact on perceived wellbeing and QoL. Fifth, themes were named in a concise and explanatory manner that captured the overall essence of the theme. Last, a report was written up providing a clear, vivid and coherent account of the analysis. It was also necessary to compare and contrast findings and to argue these findings with regards their relevance to the research question while supporting the validity of the findings by providing supporting empirical evidence relevant to the research question.

\section{Results}

From November 2017 to June 2018, 11 participants with newly diagnosed prostate cancer were recruited. Mean age was 60 SD (7) years. Seven completed the CBEP, three the HBEP and one a combination of both. Mean (standard deviation (SD) number of days between referral and starting the MedEx exercise programme was 4 (5) days. The mean (SD) duration of pre-operative exercise training was 4 (2) weeks. Adherence rates to the CBEP and HBEP (which was self-reported) was $84 \%$ and $100 \%$, respectively. All participants within the current pilot study participated in an individual interview following their participation in the community-based intervention.

Four main themes were supported by the data. Findings showed that engagement in the pre-operative exercise programme, both the CBEP and the HBEP, provided participants with: (1) a teachable moment; (2) journey of preparation; (3) a sense of optimism; and (4) a social connectedness which are reflected in the four main themes illustrated in Figure 2.

\section{(1) Teachable Moment}

Engagement in the programme acted as a teachable moment for the participants. It provided them with greater health awareness to make positive health changes. Participants expressed positive changes in a range of health behaviours including exercise, diet, alcohol consumption and lifestyle choices. The quote below alludes to the idea of "healthy mind healthy body" whereby positive physical health changes transfer into positive psychological outcomes.

"I think once that this [cancer] happens to you.... you realise the importance of exercise even if you already know that, it kind of reinforces that...it probably will have changed me that I probably try to have a better 
balance in life in terms of exercise, lifestyle, eating habits, booze etc... I would be a big believer in healthy mind healthy body, so I think yeah that....you are more regularly focused on exercise and that kicks right through to your mental state"-P5

Many of the participants developed a more holistic view to health and gained a greater awareness of the value of health. The pre-operative exercise programme acted as a catalyst for change as many of them appeared to be hopeful for their future health.

"when I came here [to the exercise programme] first - blood pressure was way up... weight was way up... probably I would've had other problems if I hadn't of come here, you know? And it has changed my life.... as regards fitness, and as regards what I eat as well, like, you know.. I was never particular about that.. I actually haven't felt so well for a long time.... continuing on the way I was, probably four or five years down the road I would be really slow... it [the exercise programme] actually has improved my quality of life now and I think it will in the future as I know what to do now. I was probably a bit careless with myself, you know"-P6

The participants said that the exercise programme was a "trigger" to make changes in order to reach an optimal level of health.

"It's probably made me think more about, the things that I should be doing, to look after myself. So maybe after this I'll keep the gym going.... It's kind of a little trigger that's gone off again" -P2

Participants expressed concern about their age in relation to the increased need to be more health conscious. The exercise programme reminded them to make more positive health changes in life:

"I'd be very conscious that it [the exercise programme] would help me through the surgery of course, but... your health in general, just heightened awareness, just how important it is, particularly in the age profile I am, that health is key."-P9

\section{(2) Journey of preparation}

Many of the participants described the pre-operative exercise programme as a stepping stone on the road to ultimate recovery. There was a notable awareness of the physical health benefits of exercise and how this contributed to psychological preparedness for surgery:

"Well I think it helps you to get over that bridge fast that's why I'm doing it [the exercise programme]. If you're fit and you're in for an operation and you're fairly fit which I would be... I think it eh... whatever keeps the mindset that I'm well able for this and that I can do it"- $P 9$

For some participants, the pre-operative exercise programme kept them "busy" giving them something to focus on and prepare for in the lead up to surgery:

"yeah it's kind of kept me, kept my brain busy as well, so I'm not thinking about the future and what I have to deal with, and more dealing with at present and trying to get myself ready for the op". - P7 
Whilst other participants described the exercise programme as a mechanism to manage stress throughout their cancer journey and prepare themselves psychologically. The participants portrayed exercise as a mode of release to escape the difficulties they are faced with:

"It's given myself my own bit of time ehm and maybe you can think of things when you're exercising but you can also when you're exercising deal with any frustrations that you may have or any difficulties you may have because you can put it out through exercise"-P10

\section{(3) Sense of Optimism}

Participation in the pre-operative exercise programme cultivated a positive attitude. This sense of optimism was a feature of the participants' psychological wellbeing which was enhanced through participation in the programme. The participants seemed to understand the value of fostering and maintaining a sense of positivity during their preparation for surgery which was beneficial to their QoL;

"I think that exercise has been the most positive part of all this. At the beginning I felt hopeless. But coming to the programme, I feel without MedEx I wouldn't be as far as I am psychologically or holistically"-P1

Some participants expressed that they found comfort in interacting with others in the group setting who were "worse off" which in turn aided their development of a heightened sense of optimism:

"yeah it [the exercise programme] has benefited me psychologically, because like at the beginning I was like, oh why me? You know... but there is people with worse cancers and worse off than me, so I think, you know, I can do this, I'll get over this and I'll move on with my life"-P2

Participation in the pre-operative exercise programme provided an overall greater appreciation for the importance of maintaining and optimising one's psychological health.

"getting the prostate cancer sort of makes you think eh where you're going in your life and how did you get it now l'm not saying any particular issue caused it but certainly a little bit more exercise maybe a little bit better diet... a little better various things in life... I also find eh I cope with things better now em because my mind is more alert and eh so eh yeh there the things that have really changed for me"- $P 8$

\section{(4) Social connectedness}

Participants gained great value from people sharing their stories and experiences of the cancer journey. A strong sense of comradery and solidarity was fostered amongst participants through their shared experience. Participants valued practical advice and support in relation to their pre-operative journey from peers with their lived cancer experience. This theme illustrates the value and usefulness of peer learning and sharing of experience through social connection rather than focusing solely on health care professional advice, research and 'academic' information 
"people [fellow participants] are so willing to share with you their stories, and tell you, you know that's not good, this is good. Like l'd be a big believer in research but it's absolutely like having a practical on top of an academic, so that really is very useful"--P1

For the participants, the friendly and patient-centred atmosphere at the CBEP contributed to the sense of wellbeing and particularly their sense of social integration. Interestingly, the social group within the exercise programme seemed to provide motivation within the participants 'journey of preparation' and highlight personal 'teachable moments';

"meeting other people with similar experiences and it's good to talk to them and see how well they are and how much they are enjoying the fitness [from taking part in the programme]"-P9

\section{Discussion}

This study demonstrated that the community-based pre-operative exercise training, delivered over a relatively short time window, enhanced perceived wellbeing and QoL in people with newly diagnosed prostate cancer facing major invasive surgery. Our findings demonstrate that participation in the exercise programme provided participants with a teachable moment, a journey of preparation, provided them with a sense of optimism and social connectedness in lead up to surgery.

The pre-operative exercise programme provided participants with a concept often described as the "teachable moment", i.e., an increased desire, willingness or capacity for change, which influences long term health and wellbeing [17]. The exercise programme fostered a holistic concept of health i.e., gave rise to greater health awareness and appeared to be a trigger to make changes in order to reach an optimal level of health. Although little has been reported in newly diagnosed prostate cancer, the "teachable moment" has been described as a promising approach for clinicians to discuss behaviour change with patients efficiently and effectively in primary care [18]. It is interesting to note that in our study, it was the consultant surgeon at cancer diagnosis who initiated the conversation about the exercise programme and that this may have positively influenced participants' health behaviours.

The pre-operative exercise programme acted as a stepping stone in the journey of preparation towards the crucial step of surgery in their road to recovery. It also provided participants with a sense of purpose and optimism. This may be important as the time before major surgery is associated with psychological stress, fear and anxiety [19]. Previous research has showed that physical activity is a promising strategy for cancer survivors as it provides an opportunity to focus on health rather than illness [8]. Active modes of control such as engaging in an exercise programme or adopting a healthier diet have been shown to be the most effective method of enhancing QoL when faced with a life-threatening illness [20]. We showed that the exercise programme encouraged participants to adopt a proactive approach while awaiting surgery, which in turn, boosted their perceived wellbeing

The sense of comradery and solidarity that was fostered amongst participants during the programme acted as an avenue of social support and was identified as one of the key contributors to the participants' 
wellbeing. This social connection with peers facilitated sharing of individual lived experience of the preoperative journey beyond 'academic' or 'research' information. Social networks and relationships as well as the social environment have been highlighted as being important aspects of the cancer care journey [21]. In the current study, sharing stories about the cancer journey fostered a sense of connectedness amongst participants (note: participants on the HBEP attended MedEx at the same time as the CBEP and were integrated within the group). Similar effects have been reported in a study among women with breast cancer facing similar challenges where the importance of mutual understanding and support was documented [22-23]. Specific to people with prostate cancer, exercise interventions have been shown to reduce both psychological and social health problems as they combat the emasculating effects of prostate cancer treatment and they provide an element of social support [24]. It is possible that the social aspect of the exercise programme led participants to adopt a supportive social network which may have contributed positively to their perceived social wellbeing. Our study findings highlight the importance of relationships, social support and networking with peers among people with newly diagnosed prostate cancer while awaiting surgery. Interestingly, participants who engaged in the HBEP did not report lower levels of social connectedness in comparison to participants within the CBEP. This finding requires further exploration in future research to understand the elements of a community-based intervention which facilitate social connection.

Findings from our study are timely following two recent reports published in 2018 on the important role exercise plays in the cancer journey [25-26]. The Clinical Oncology Society of Australia published recommendations that exercise should be embedded as part of standard practice in cancer care and to be viewed as an adjunct therapy that helps counteract the adverse effects of cancer and its treatment [25]. Pre-operative clinical guidelines and recommendations on exercise training have also been recently published that provide practical guidance for providing safe and effective exercise [26]. However, specific to Ireland, it has been recently reported that healthcare professionals need significant education in improving their knowledge on physical activity programmes in cancer [27]. Pre-operative exercise programmes can help to bridge the gap between the lack of specialist knowledge from healthcare professionals regarding cancer care and exercise by providing the knowledge and skills that can aid people with cancer to live more active and healthier lives. Our findings highlight the importance of a preoperative exercise programme delivered alongside routine care for people with newly diagnosed prostate cancer.

\section{Strengths and limitations}

Strengths of this work include the novelty of the community-based model which is a more accessible, scalable and sustainable alternative to hospital-based programmes. Additionally, the rapid referral and access from the local hospital to the community, the inclusion of a homogenous cancer type and treatment pathway. The exercise programme was delivered alongside standard routine care in the time window between diagnosis and surgery. 
Limitations include the small sample size due to the nature of the pilot study. Additionally, participants in this study had little comorbidity with a reasonable level of functioning therefore it is possible they were a motivated group which increases the risk of selection bias. Furthermore, the dose-response relationship was not explored, which may have had negative implications for the results as some participants may have had more/less time to derive affect from the programme. The semi-structured interviews were carried out by a research team member who was co-ordinating the exercise sessions (LL), however a number of randomly chosen interviews were also conducted by a research team member who was not directly involved in the exercise sessions (RMcG) in an effort to limit potential bias within interviews.

\section{Conclusion}

This community-based pre-operative exercise programme provided people with newly diagnosed prostate cancer with a teachable moment, a journey of preparation, a sense of optimism and social connectedness, all of which contributed to enhanced wellbeing and QoL. These four main themes give valuable insight into the complex demands of the pre-operative journey and the positive role that preoperative exercise interventions may play in the perceived well-being and QoL of men with newly diagnosed cancer. These data highlight the requirement for future work to explore the effect of preoperative exercise interventions on wellbeing and QoL in people with newly diagnosed cancer in a larger sample.

\section{Declarations}

\section{Ethics approval and consent to participate}

The study was approved by Dublin City University Research Ethics Committee (REC/2015/207).

\section{Consent for publication}

Not applicable.

\section{Availability of data and materials}

The datasets used and/or analysed during the current study are available from the corresponding author on reasonable request.

\section{Competing interests}

The authors declare they have no competing interests.

\section{Funding}

None

\section{Authors' contributions}


LL, KOM, BF and NMcC conceived the study. LL, BF and NMcC contributed to study design. LL undertook data collection. DW and RMcG undertook the statistical analysis. LL drafted the manuscript which underwent revision by all other authors. All authors read and approved the final manuscript.

\section{Acknowledgements}

To all who participated in the study

\section{Abbreviations}

CBEP - centre-based exercise programme

FITT-P - frequency, intensity, time, time - progression

HBEP - home-based exercise programme

HR - health related

HRQoL - health related quality of life

QoL - quality of life

RPE - rate of perceived exertion

\section{References}

1. Pearce RM, Grocott MPW. Managing perioperative risk in patients undergoing elective non-cardiac surgery. BMJ. 2011; doi: 10.1136/bmj.d5759.

2. Moonesinghe SR, Harris S, Mythen MG, et al. Survival after postoperative morbidity: a longitudinal observational cohort study. Br J Anaest. 2014;113 (6):977-984.

3. Older P, Smith R, Courtney $P$, et al. Preoperative evaluation of cardiac failure and ischemia in elderly patients by cardiopulmonary exercise testing. Chest. 1993; 104 (3):701-704.

4. Hartley RA, Pichel AC, Grant SW, et al. Preoperative cardiopulmonary exercise testing and risk of early mortality following abdominal aortic aneurysm repair. Br J Surg. 2012;99 (11):1539-1546.

5. Prentis JM, Manas DM, Trenell MI, et al. Submaximal cardiopulmonary exercise testing predicts 90day survival after liver transplantation. Liver Transpl. 2012;18 (2):152-159.

6. West MA, Asher R, Browning M, et al. Validation of preoperative cardiopulmonary exercise testingderived variables to predict in-hospital morbidity after major colorectal surgery. Br J Surg. 2016;103(6):744-752.

7. Thompson AR, Peters N, Lovegrove RE, et al. Cardiopulmonary exercise testing provides a predictive tool for early and late outcomes in abdominal aortic aneurysm patients. Ann R Coll Surg Engl. 2011;93 (6):474-481. 
8. Hennis PJ, Meale PM, Hurst RA, et al. Cardiopulmonary exercise testing predicts postoperative outcome in patients undergoing gastric bypass surgery. Br J Anaest. 2012;109(4):566-571.

9. Snowden CP, Prentis JM, Aderson HL, et al. Submaximal cardiopulmonary exercise testing predicts complications and hospital length of stay in patients undergoing major elective surgery. Ann Surg. 2010;251(3):535-541.

10. Cormie, P, Atkinson M, Bucci L, et al. Clinical oncology society of Australia position statement on exercise in cancer care. Med J Aust. 2019;210(1):54-54.

11. Mujovic N, Subotic D, Marinkovic M, et al. Preoperative pulmonary rehabilitation in patients with nonsmall cell lung cancer and chronic obstructive pulmonary disease. Arch Med Sci. 2014;10 (1):68-75.

12. West MA, Loughney L, Lythgoe $D$, et al. Effect of prehabilitation on objectively measured physical fitness after neoadjuvant treatment in preoperative rectal cancer patients: a blinded interventional pilot study. Br J Anaest. 2014;114 (2):244-251.

13. Barakat HM, Shahin Y, Barnes R, et al. Supervised exercise program improves aerobic fitness in patients awaiting abdominal aortic aneurysm repair. Ann Vasc Surg. 2014;28 (1):74-79.

14. Coats V, Maltais F, Simard S, et al. Feasibility and effectiveness of a home-based exercise training program before lung resection surgery. Can Respir J. 2013;20(2):e10-6.

15. Gillis C, Li C, Lee L, et al. Prehabilitation versus Rehabilitation; A Randomized Control Trial in Patients Undergoing Colorectal Resection for Cancer. Anesthesiology. 2014;121(5):937-947.

16. Awasthi R, Minnella EM, Ferreira V, et al. Supervised exercisetraining with multimodal pre-habilitation leads to earlier functional recovery following colorectal cancer Acta Anesthesiol Scand. 2018; doi: 10.1111/aas.13292.

17. World Health Organization . Interventions on Diet and Physical Activity: What works: Summary Report. World Health Organization; Geneva, Switzerland: 2009

18. Salbach NM, Howe JA, Brunton K, Sailsbury K, Bodiam L. Partnering to increase access to community exercise programs for people with stroke, acquired brain injury, and multiple sclerosis. $J$ Phys Act Health 2014;11:838-45.

19. Smith C, Olson K, Hale LA, Baxter D, Schneiders AG. How does fatigue influence community-based exercise participation in people with multiple sclerosis? Disabil Rehabil 2011;33:2362-71.

20. Stuart M, Benvenuti F, Macko R, Taviani A, Segenni L, Mayer F et al. Community-based adaptive physical activity program for chronic stroke: feasibility, safety, and efficacy of the Empoli Model. Neurorehabil Neural Repair 2009;23:726-34.

21. Rao R, Cruz V, Peng Y, et al. Bootcamp during neoadjuvant chemotherapy for breast cancer: a randomized pilot trial. Breast Cancer. 2012; 6:39-46.

22. Singh F, Newton RU, Baker MK, et al. Feasibility of presurgical exercise in men with prostate cancer undergoing prostatectomy. Integr Cancer Ther. 2017;16 (3): 290-299.

23. ATS/ACCP. American Thoracic Society and American College of Chest Physicians Statement on cardiopulmonary exercise testing. Am J Respir Crit Care Med. 2003;167(2):211-277. 
24. Website URL link: https://www.dcu.ie/shhp/Medex-Newsletter.shtml.

25. Boereboom CL, Phillips BE, Williams JP, et al. A 31-day time to surgery compliant exercise training programme improves aerobic health in the elderly. Tech Coloproctol. 2016;20 (6):375-382.

26. Rosales W, Cofre C, Alejandra C, Bertona C, Vizcaya A, Gonzalez J, Bajuk J, Rodriguez M. Validation Of the Borg scale in participants with type 2 diabetes mellitus. Rev Med Chill 2016; 144 (9): 11591163.

27. HollandAE, Spruit MA, Troosters T, et al. An official European Respiratory Society/American Thoracic Society technical standard: field walking tests in chronic respiratory disease (Task Force Report). Eur Resp J. 2014;44: 1428-1466.

28. Mathiowetz V, Weber K, Volland G, et al. Reliability and validity of grip and pinch strength evaluations. J Hand Surg Am. 1984;9(2): 222-226.

29. Ozalevli S, Ozden A, Akkoclu A. Comparison of the Sit-to-Stand Test with 6 min walk test in patients with chronic obstructive pulmonary disease. Respir Med. 2007;101(2):286-293.

30. Aaronson NK, Ahmedzai S, Bergman B, et al. The European Organization for Research and Treatment of Cancer QLQ-C30: a quality-of-life instrument for use in international clinical trials in oncology. $J$ Natl Cancer Inst. 1993;85:365-376.

31. Rabin R, de Charro F. EQ-5D: a measure of health status from the EuroQol Group. Ann Med. 2001;33:337-343.

32. Tew GA, Ayyash R, Durrand J, et al. Clinical guideline and recommendations on pre-operative exercise training in patients awaiting major non-cardiac surgery. Anaesthesia. 2018; doi: 10. 111/anae.14177

33. Carli F, Charlebois $P$, Stein $B$, et al. Randomized clinical trial of prehabilitation in colorectal surgery. $\mathrm{Br}$ J Surg. 2010;97(8):1187-1197.

34. Kim DJ, Mayo NE, Carli F, et al. Responsive Measures of Prehabilitation in Patients Undergoing Bowel Resection Surgery. Tohoku J Exp Med. 2009;217:109-115.

35. Morielli AR, Usmani N, Boule NG, et al. A Phase I Study Examining the Feasibility and Safety of an Aerobic Exercise Intervention in Patients With Rectal Cancer During and After Neoadjuvant Chemoradiotherapy. Oncol Nurs Forum. 2016;43(3):352-362.

36. Kothmann E, Batterham AM, Owen SJ, et al. Effect of short-term exercise training on aerobic fitness in patients with abdominal aortic aneurysms: a pilot study. Br J Anaest. 2009;103(4):505-510.

37. Thederan I, Chandrasekar T, Tennstedt P, et al. Poor Adherence to International CancerPrevention Recommendations Among Patients With Prostate Cancer: First Results From the MARTINI-Lifestyle Cohort. Eur Urol Focus. 2019; doi: 10.1016/j.euf.2019.01.006

38. Mavros MN, Athanasiou S, Gkegkes IA, et al. Do psychologocial variables affect early surgical recovery? 2011; doi.org/10.1371/journal.pone.0020306.

39. Crandall K, Maguire R, Campbell A, et al. qualitative study exploring the views, attitudes and beliefs of patients and health professionals towards exercise intervention for people who are surgically treated for lung cancer. Eur J Cancer Care. 2019;27(2):e12828. 
Table 1. Exercise training prescription described using the FITT principle (frequency, intensity, time, type) and progression

\begin{tabular}{|c|c|}
\hline Frequency & $\begin{array}{l}\text { 3-5 sessions per week depending on the time interval before surgery (i.e. } \\
\text { if patients had a } 2 \text {-week interval, they were advised to undertake } 5 \\
\text { sessions per week or }>2 \text { weeks, they were advised to undertake } \geq 3 \\
\text { sessions per week). }\end{array}$ \\
\hline Intensity & $\begin{array}{l}\text { (1) Interval (moderate to high intensity) exercise training: moderate and } \\
\text { high intensities, derived based on RPE scale (13: somewhat hard and 15: } \\
\text { hard) using the same concept as West et al., } 2014 \text { without the use of } \\
\text { CPET to inform intensities. } \\
\text { ( } 2 \text { ) High intensity exercise training: derived based on RPE scale (16: hard } \\
\text { to very hard) using the same concept as Boereboom et al., } 2016 \text { but } \\
\text { without the use of CPET. } \\
\text { (3) Resistance training: included performing } 3 \text { sets x } 12 \text { repetitions. The } \\
\text { load was selected based on individual ability (i.e. kg of weight using } 12 \\
\text { repetition maximum (RM) with a minimum } 30 \text { sec recovery period } \\
\text { between each set. }\end{array}$ \\
\hline Time & $\begin{array}{l}\text { Total: ranged between } 40 \text { to } 60 \text { mins } \\
\text { "(1) The first interval (moderate to high intensity) exercise session was } \\
30 \text { minutes: } 5 \text { minute warm-up followed by } 4 \text { repeated bouts of moderate } \\
\text { intensity ( } 3 \text { min) to high intensity ( } 2 \text { min) intervals and } 5 \text { minute cool } \\
\text { down. The second sessions onwards was } 40 \text { minutes: } 5 \text { minute warm-up } \\
\text { followed by } 6 \text { repeated bouts of moderate intensity ( } 3 \text { min) to high } \\
\text { intensity ( } 2 \text { min) intervals and } 5 \text { minute cool down. } \\
\text { (2) The high intensity exercise training was } 17.5 \text { minutes: } 2 \text { minute warm- } \\
\text { up followed by } 5 \text { repeated bouts of high intensity ( } 1 \text { min) and recovery } \\
\text { (90 seconds) intervals and a } 3 \text { minute cool down”. } \\
\text { (3) Resistance training involved completing } 3 \text { sets x } 12 \text { repetitions } \\
\text { (approx. } 20 \text { minutes). }\end{array}$ \\
\hline Type & $\begin{array}{l}\text { The CBEP aerobic exercise modalities included upright cycle ergometer; } \\
\text { recumbent cycle ergometer; treadmill; elliptical ergometer; and rowing } \\
\text { ergometer, depending on patient preference. The CBEP resistance } \\
\text { training involved a circuit of strength 8-10 stations alternating upper and } \\
\text { lower body exercises using the following machines: shoulder press; lat } \\
\text { pulldown; tricep press; squat; chest press; leg extension; hamstring curl; } \\
\text { and back row. } \\
\text { The HBEP aerobic exercise included walking; cycling; swimming; or any } \\
\text { other aerobic activity they enjoyed. The HBEP resistance training } \\
\text { involved upper and lower body exercises as per CPEB except using free } \\
\text { weights/dumb bells dependent on availability. }\end{array}$ \\
\hline Progression & $\begin{array}{l}\text { Exercise intensity (interval/high) was progressed every } 5 \text { sessions (i.e. } \\
\text { the intensity was increased by } 1 \text { level in the CBEP). } \\
\text { Resistance training progressed following completion of } 3 \text { consecutive } \\
\text { sessions comfortably (i.e. weight was increased by } 1 \mathrm{~kg} \text { ). }\end{array}$ \\
\hline
\end{tabular}




\begin{abstract}
Abbreviations: RPE (rate of perceived exertion); CPET (cardiopulmonary exercise test); CBEP (centrebased exercise programme); HBEP (home-based exercise programme). Note: The aerobic high intensity training was only prescribed to certain individuals who could tolerate the interval training very well and only to participants on the CEBP. The aerobic exercise training included interval and high intensity training, which were alternated between every second sessions.
\end{abstract}

\title{
Figures
}




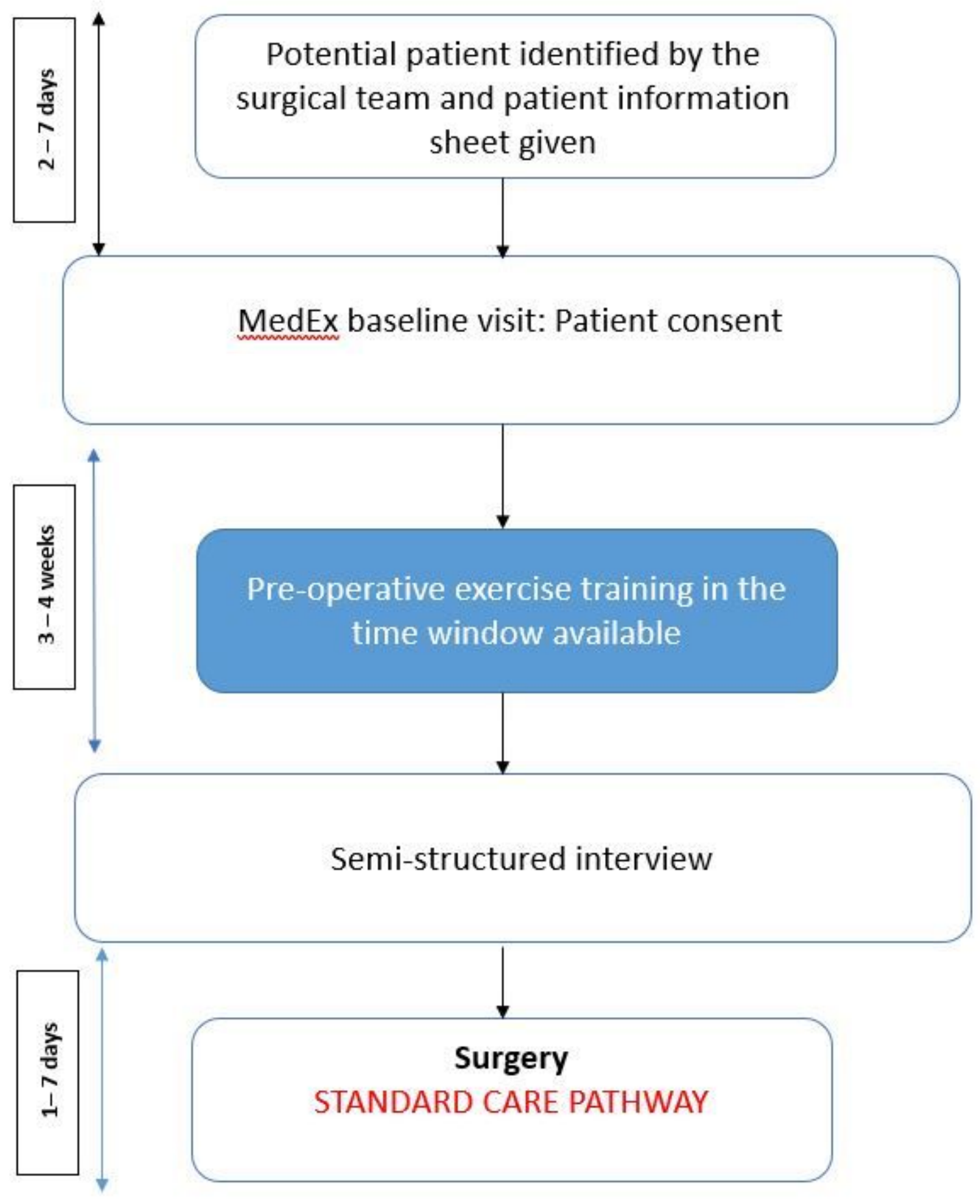

Figure 1

Study flow algorithm illustrating patient pathway 


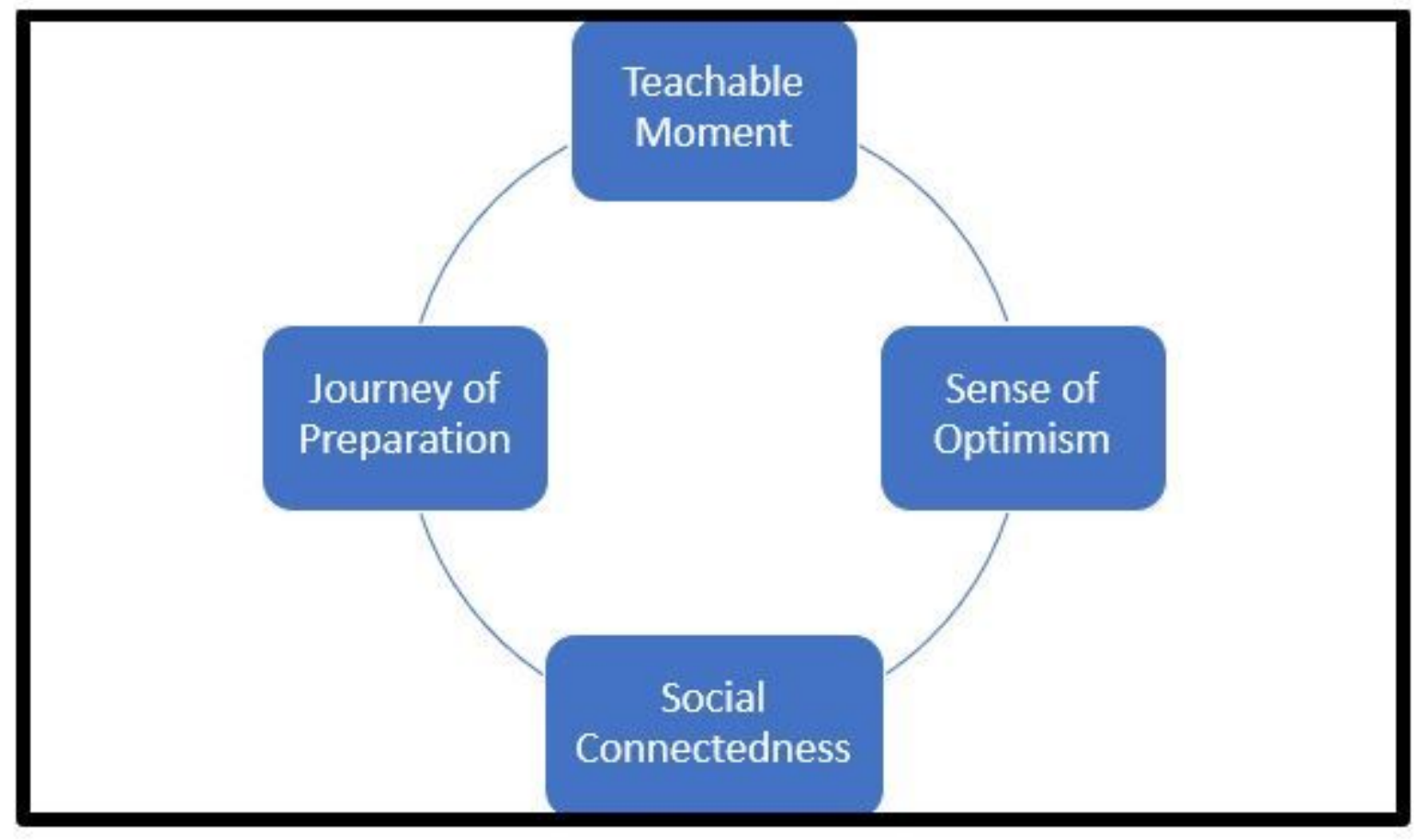

Figure 2

Illustration of main themes 